\title{
Ferromagnetic quantum critical point induced by dimer-breaking in $\mathrm{SrCo}_{2}\left(\mathrm{Ge}_{1-x} \mathrm{P}_{x}\right)_{2}$
}

\author{
Shuang Jia ${ }^{1}$, Pawina Jiramongkolchai ${ }^{1}$, M. R. Suchomel ${ }^{2}$, B. H. Toby ${ }^{2}$, J. G. Checkelsky ${ }^{3}$, N. P. Ong ${ }^{3}$ \\ and R. J. Cava ${ }^{1 \star}$
}

In contrast to classical phase transitions driven by temperature, a quantum critical point (QCP) defines a transition at zero temperature that is driven by non-thermal parameters $^{1-3}$. In the known quantum critical $d$-electron systems, tuning the electronic bandwidth by means of changing the applied pressure or unit-cell dimensions, or tuning the $d$-state population, is used to drive the criticality ${ }^{4-6}$. Here we describe how a novel chemical parameter, the breaking of bonds in Ge-Ge dimers that occurs within the intermetallic framework in $\mathrm{SrCO}_{2}\left(\mathrm{Ge}_{1-x} \mathrm{P}_{x}\right)_{2}$, results in the appearance of a ferromagnetic (FM) QCP. Although both $\mathrm{SrCO}_{2} \mathrm{P}_{2}$ and $\mathrm{SrCO}_{2} \mathrm{Ge}_{2}$ are paramagnetic, weak itinerant ferromagnetism unexpectedly develops during the course of the dimer breaking, and $a$ QCP is observed at the onset of the FM phase. The use of chemical bond breaking as a tuning parameter to induce QCP opens an avenue for designing and studying novel magnetic materials.

$\mathrm{SrCo}_{2}\left(\mathrm{Ge}_{1-x} \mathrm{P}_{x}\right)_{2}$ belongs to the layered, tetragonal $\mathrm{ThCr}_{2} \mathrm{Si}_{2}$ structure type, which has over 600 intermetallic members. This $\mathrm{AT}_{2} \mathrm{X}_{2}$ structure is formed by stacking covalently bonded transition metal-metalloid $\mathrm{T}_{2} \mathrm{X}_{2}$ layers, made from edge-sharing $\mathrm{TX}_{4}$ tetrahedra, with ionic A atoms (see Fig. 1a inset). These compounds have attracted recent interest owing to the rich physics they exhibit, such as non-Fermi-liquid behaviour in $\mathrm{YbRh}_{2} \mathrm{Si}_{2}$ (ref. 7) and unconventional superconductivity in $\mathrm{K}$-doped $\mathrm{BaFe}_{2} \mathrm{As}_{2}$ (ref. 8). The existence of a molecule-like $\mathrm{X}-\mathrm{X}$ dimer that crosses the $\mathrm{A}$ atom layer in certain members of this family was noted 20 years ago, providing an explanation for their anomalously low ratio of stacking to in-plane lattice parameters ${ }^{9,10}(c / a)$. This X-X bonding between neighbouring $\mathrm{T}_{2} \mathrm{X}_{2}$ layers pulls the layers closer together and induces a relaxation of the in-plane lattice dimension, leading to what are known as collapsed tetragonal (cT) cells. In contrast, the absence of an $\mathrm{X}-\mathrm{X}$ dimer results in uncollapsed tetragonal (ucT) cells. The lack of an interlayer X-X bond substantially weakens the electronic interactions between neighbouring $\mathrm{T}_{2} \mathrm{X}_{2}$ layers, leading to less three-dimensional (3D)-like electronic structures and Fermi surface topologies for the ucT phases compared with the cT phases ${ }^{11-13}$. Lattice collapse transitions from ucT to cT phases driven by applied pressure or chemical pressure were not shown to have a great impact on the physical properties of the compounds until recently ${ }^{14-18}$.

$\mathrm{SrCo}_{2} \mathrm{Ge}_{2}$ is a typical cT phase, with a $c / a$ ratio of 2.6 due to the presence of a strong interlayer $\mathrm{Ge}-\mathrm{Ge}$ bond, whereas $\mathrm{SrCo}_{2} \mathrm{P}_{2}$, with a $c / a$ ratio of 3.1 , is a typical ucT phase. The solid solution of these two compounds should therefore trace the crossover from cT to ucT phases, and, as shown in Fig. 1 a, the $\operatorname{SrCo}_{2}\left(\mathrm{Ge}_{1-x} \mathrm{P}_{x}\right)_{2}$ solid solution shows anomalous $\mathcal{S}$-shape changes in both $a$ and $c$ as a function of $x$. Although $a$ and $c$ vary noticeably, the absence of a region of chemical phase separation indicates that the transition from cT to ucT is continuous in nature. Figure $1 \mathrm{~b}$, which plots important interatomic distances and bond angles derived from structural refinements, shows that the $\mathcal{S}$-shape behaviour of $a$ and $c$ arises from the breaking of the $\mathrm{X}-\mathrm{X}$ dimer on substitution of $\mathrm{P}$ for Ge, which begins at $x \sim 0.4$ and is completed by $x \sim 0.8$. Therefore, the structural phase diagram consists of a cT phase for $x<0.4$, a ucT phase for $x>0.8$ and an intermediate state for $0.4<x<0.8$. The $\mathrm{X}-\mathrm{X}$ bond length within the dimer varies from $2.9 \AA$ for $x=0$ to $3.4 \AA$ for $x=1$, crossing from bonded to non-bonded distances ${ }^{9,10}$. Correspondingly, the Co-Co separation within the $\mathrm{T}_{2} \mathrm{X}_{2}$ layers decreases as the layers separate as a result of the dimer breaking, increasing the in-plane $\mathrm{Co}-\mathrm{Co}$ interactions. The size and shape of the $\mathrm{CoX}_{4}$ tetrahedron (the Co-X distances and Co-X-Co tetrahedral angle in Fig. 1b) also vary considerably in the crossover region, $0.4 \leq x \leq 0.8$, as the weakening of the $\mathrm{X}-\mathrm{X}$ bond allows the structure to relax to a state dominated by $2 \mathrm{D}$ rather than $3 \mathrm{D}$ bonding interactions. The crystal structure characterization therefore implies that although the electronic structures should be quite different for the $\mathrm{cT} \mathrm{SrCo}_{2} \mathrm{Ge}_{2}$ and ucT $\mathrm{SrCo}_{2} \mathrm{P}_{2}$ phases, the properties of the solid solution should vary continuously between the two endmembers.

As shown in Fig. 2, the magnetic properties of $\operatorname{SrCo}_{2}\left(\mathrm{Ge}_{1-x} \mathrm{P}_{x}\right)_{2}$ do not follow the simple trend implied by the structural variation. Both parent compounds, $\mathrm{SrCo}_{2} \mathrm{Ge}_{2}$ and $\mathrm{SrCo}_{2} \mathrm{P}_{2}$, are Pauli paramagnetic (PM), although the latter compound is Stonerenhanced. These results are consistent with previous studies ${ }^{15,18,19}$. A highly unexpected FM state appears, however, for $0.35 \leq x \leq 0.7$ (Fig. $2 \mathrm{a}$ and b). As $x$ increases from 0 towards 1 , the series exhibits spontaneous magnetization, which first appears at $2 \mathrm{~K}$ with a small saturated moment $\left(\mu_{\mathrm{sat}}=0.02 \mu_{\mathrm{B}} / \mathrm{Co}\right)$ for $x=0.35$. This $\mu_{\text {sat }}$ increases to a maximum value $\left(0.1 \mu_{\mathrm{B}} / \mathrm{Co}\right)$ at $x=0.55$ before decreasing again (see Fig. 3 ). For $x>0.8$, the FM ground state disappears entirely.

The FM ordering temperature $\left(T_{\mathrm{C}}\right)$ was determined from the temperature-dependent magnetization and magnetization isotherm (Arrott plot ${ }^{20}$ measurements (Fig. 2c). As shown in Fig. 4, $T_{\mathrm{C}}$ varies in the same manner as $\mu_{\text {sat }}$ : the highest $T_{\mathrm{C}}$ is $35 \mathrm{~K}$ for $x=0.55$. Figure $2 \mathrm{~d}$ shows that the series exhibits high-temperature Curie-Weiss behaviour for $x \geq 0.35$. The effective moment $\left(\mu_{\text {eff }}\right)$ increases from $0.64 \mu_{\mathrm{B}} /$ Co for $x=0.35$ to $1.7 \mu_{\mathrm{B}} /$ Co for $x=1$. These $\mu_{\text {eff }}$ values are 10-30 times larger than the corresponding $\mu_{\text {sat }}$ values, placing these FM compounds solidly in the weak itinerant FM region of the Rhodes-Wohlfarth plot ${ }^{21}$.

To understand how the system evolves from Pauli PM to FM, and then back to enhanced PM with increasing $x$, the samples near

${ }^{1}$ Department of Chemistry, Princeton University, Princeton, New Jersey 08544, USA, ${ }^{2}$ Argonne National Laboratory, Advanced Photon Source, Argonne Illinois 60439, USA, ${ }^{3}$ Department of Physics, Princeton University, Princeton, New Jersey 08544, USA. *e-mail: rcava@princeton.edu. 


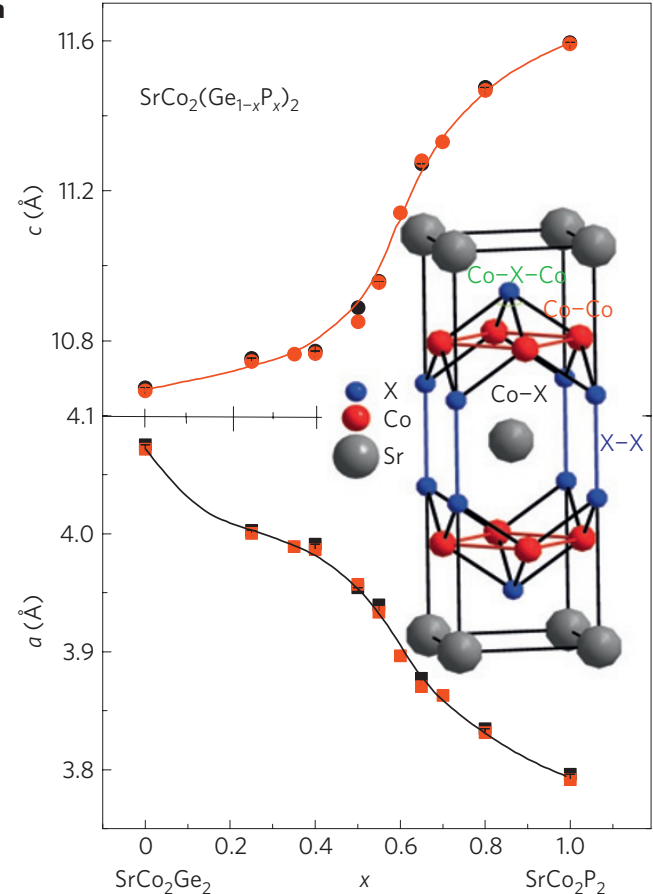

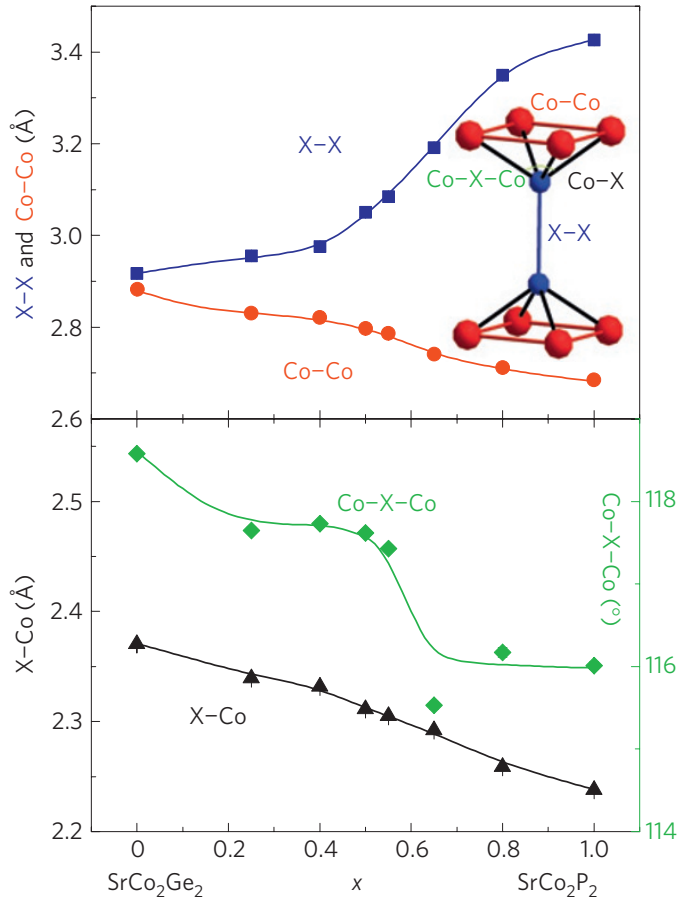

Figure 1 | Lattice collapse transition in $\mathrm{SrCo}_{2}\left(\mathrm{Ge}_{1-x} \mathrm{P}_{\mathbf{X}}\right)_{2}$. $\mathbf{a}$, Lattice parameters. Red symbols: laboratory X-ray diffraction (XRD); black symbols: synchrotron XRD. Inset: Unit cell for $\mathrm{SrCo}_{2} \mathrm{X}$. $\mathbf{b}, \mathrm{X}-\mathrm{X}, \mathrm{Co}-\mathrm{Co}$ and $\mathrm{X}-\mathrm{Co}$ bond lengths, and the $\mathrm{Co}-\mathrm{X}-\mathrm{Co}$ angle, from synchrotron XRD. All the error bars are statistical errors from the GSAS refinement.
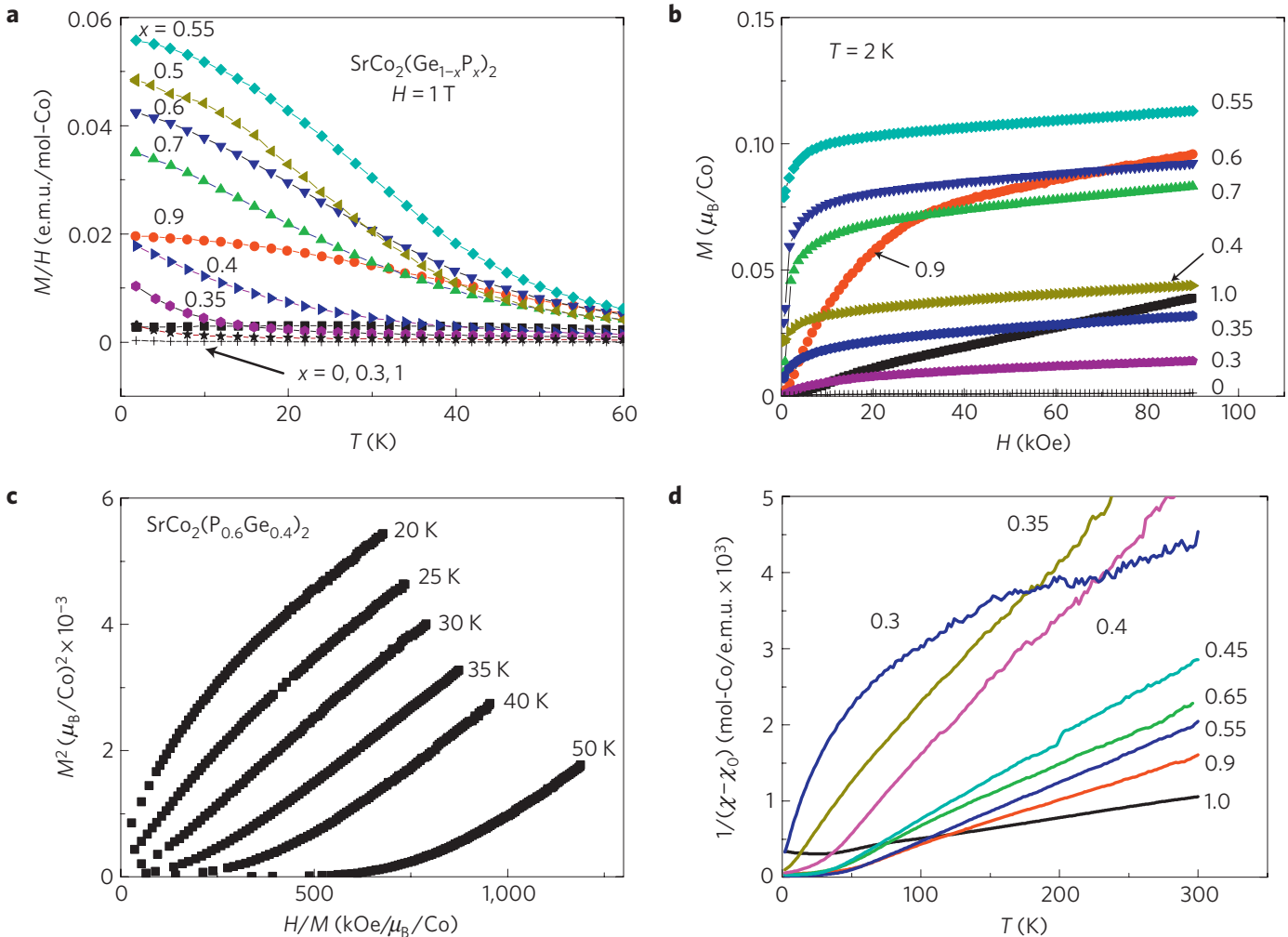

d

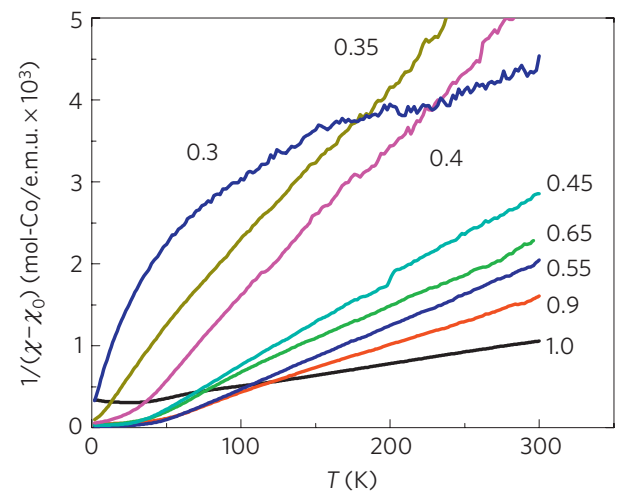

Figure 2 | The magnetic properties of $\mathrm{SrCo}_{2}\left(\mathrm{Ge}_{1-x} \mathbf{P}_{x}\right)_{2} \cdot \mathbf{a}, M / H$ versus T. $\mathbf{b}, M$ versus $H$ at $2 \mathrm{~K}$. $\mathbf{c}$, Arrott plot for $x=0.6$. d, Curie-Weiss behaviour for $x \geq 0.35$.

the critical region were measured in more detail. Figure $2 \mathrm{a}, \mathrm{b}$ shows that the FM ground state develops as $x$ increases from 0.3 to 0.4 . The Arrott plot measurements show that $T_{\mathrm{C}}$ for $x=0.35$ (Fig. 3a) and 0.4 (data not shown) are $2 \pm 0.2 \mathrm{~K}$ and $12 \pm 1 \mathrm{~K}$ respectively.
Figure $3 \mathrm{~b}$ shows the low-temperature magnetic susceptibility $(\chi)$ plotted with respect to $T^{-4 / 3}$ for $x=0.3,0.325$ and 0.35 . Although the $\chi$ values are highly sensitive to $x$, the sample with $x=0.325$ exhibits very close to the power-law behaviour expected near a FM 
a
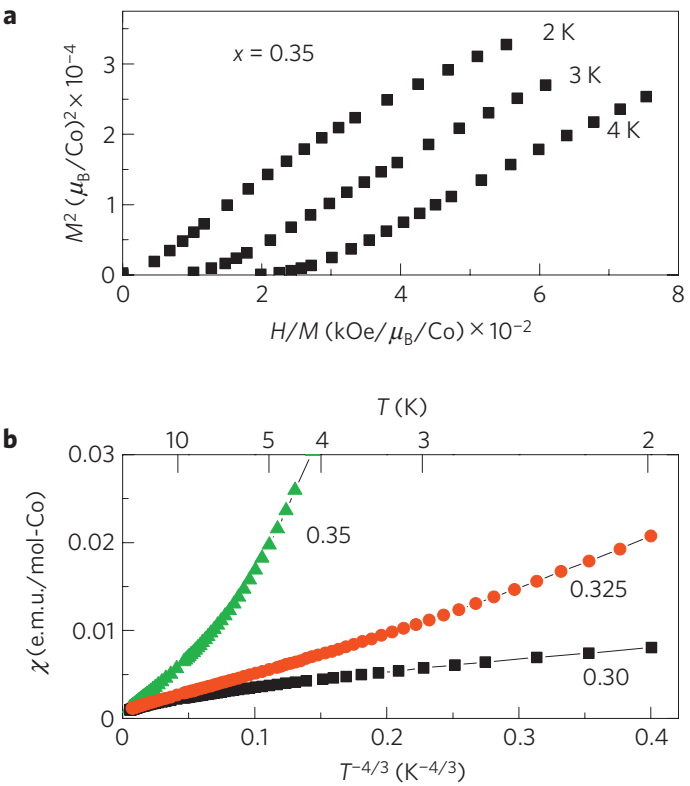

c

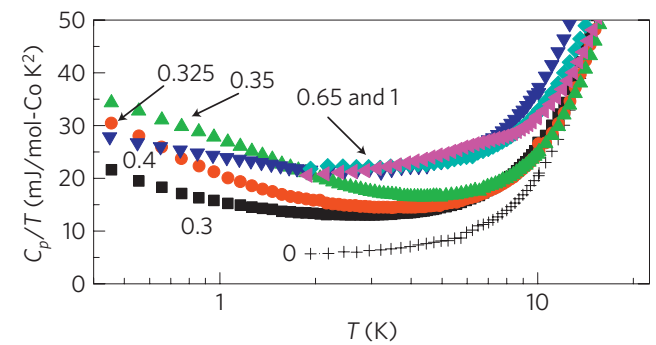

Figure 3 | QCP behaviour near $\mathbf{x}=\mathbf{0 . 3 2 5}$. $\mathbf{a}$, Arrott plot for $x=0.35$ showing that $T_{\mathrm{C}}$ is lower than $2 \mathrm{~K} . \mathbf{b}, \chi$ versus $T^{-4 / 3}$ for $x=0.30,0.325$ and 0.35 showing a nearly linear dependence at $x=0.325$. c, Low-temperature heat capacity for $x=0,0.3,0.325,0.35,0.4,0.65$ and 1 showing $\log T$ divergence near $x=0.325$.

QCP (ref. 1). Figure 3c shows the heat-capacity data as $C_{p} / T$ versus $T$ on semi-logarithmic axes. Initially, $C_{p} / T$ is a constant at low temperatures. However, at $x=0.325$ and $0.35, C_{p} / T$ exhibit clear logarithmic behaviour with respect to $T$, which is characteristic of a system approaching a FM QCP (ref. 22). When $x \geq 0.4$, the logarithmic $C_{p} / T$ behaviour is suppressed and Fermi-liquid behaviour $\left(C_{p} / T=\gamma_{0}+\beta T^{2}\right)$ is recovered. There are no signs of spin-glass behaviour ${ }^{23}$ in any of the magnetization or heat capacity data near $x=0.35$. All these results demonstrate the presence of a FM QCP driven by $x$ near $x=0.325$ in $\operatorname{SrCo}_{2}\left(\mathrm{Ge}_{1-x} \mathrm{P}_{x}\right)_{2}$.

In contrast to the QCP near $x=0.325$, a simple crossover from the FM state to an enhanced PM state appears for $x \sim 0.8$. Figure $4 \mathrm{~b}$ shows that $T_{\mathrm{C}}$ maintains a large value $(20 \pm 2 \mathrm{~K})$ for $x=0.7$. When $x>0.7$, the magnetization remains large but the spontaneous magnetization disappears (Fig. 2a,b). Correspondingly, the heat capacity data show Fermiliquid behaviour with invariant $\gamma_{0}$ for $0.5 \leq x \leq 1$.

As summarized in Fig. 4, the physical properties of $\mathrm{SrCo}_{2}\left(\mathrm{Ge}_{1-x} \mathrm{P}_{x}\right)_{2}$ are strongly correlated with its variation in structure. When $x$ increases, both the zero-temperature susceptibility $\left(\chi_{T=0}\right)$ and $\gamma_{0}$ increase, but the QCP occurs exactly when the $\mathrm{X}-\mathrm{X}$ dimer is beginning to break (Fig. $4 \mathrm{~b}$ ). Furthermore, the FM ground state appears only during the breaking of the dimer. When the dimer is fully broken, the FM state disappears and $\chi_{T=0}$ decreases with increasing $x$, whereas $\gamma_{0}$ is invariant. The phase diagram in Fig. $4 \mathrm{~b}$ clearly demonstrates that the QCP and ferromagnetism are not induced by a simple electron doping

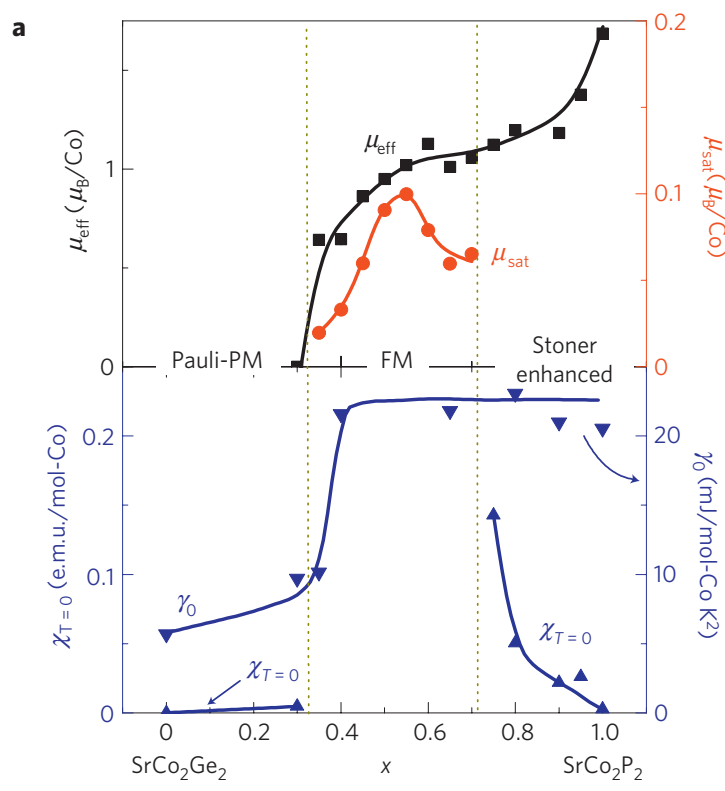

b

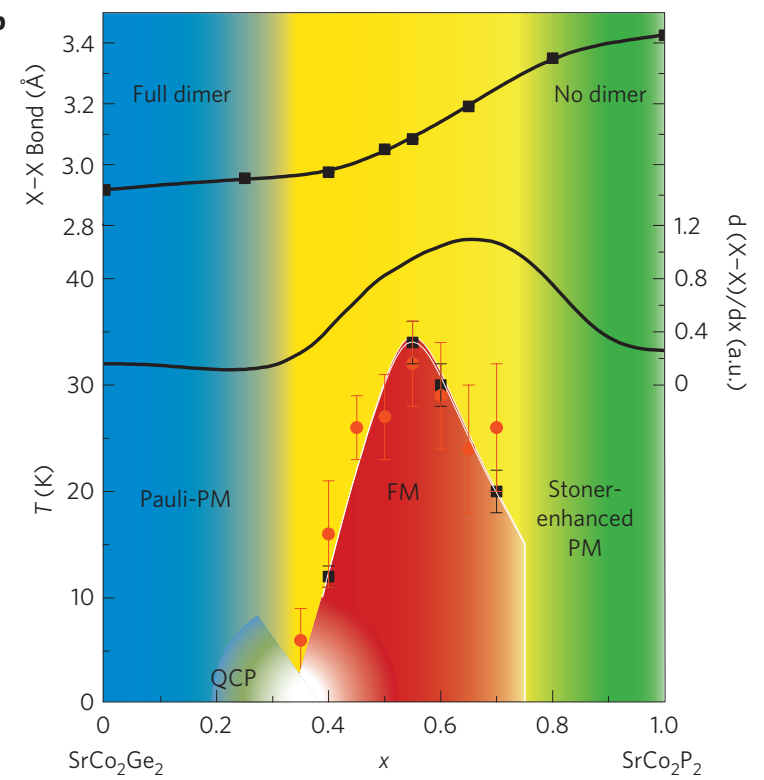

Figure 4 | Summary and phase diagram for $\mathrm{SrCo}_{2}\left(\mathrm{Ge}_{1-x} \mathbf{P}_{\mathbf{x}}\right)_{2}$. a, Physical properties for $\mathrm{SrCO}_{2}\left(\mathrm{Ge}_{1-x} \mathrm{P}_{x}\right)_{2}$. Upper panel: effective moment and saturated moment; lower panel: zero-temperature susceptibility $\left(\chi_{T}=0\right)$ and Sommerfeld coefficient $\left(\gamma_{0}\right)$. b. The structural and magnetic phase diagram for $\mathrm{SrCO}_{2}\left(\mathrm{Ge}_{1-x} \mathrm{P}_{x}\right)_{2}$. Black symbols: $T_{\mathrm{C}}$ determined by the Arrott plot; the error bars are the temperature interval of the measured isotherms. Red symbols: $T_{C}$ determined by assuming $M\left(T_{C}\right)=20 \pm 10 \% M(2 \mathrm{~K})$.

effect, but rather by the breaking of the dimer. This result is consistent with continuing studies on $\mathrm{CaCo}_{2}\left(\mathrm{Ge}_{1-x} \mathrm{P}_{x}\right)_{2}$ and $\mathrm{BaCo}_{2}\left(\mathrm{Ge}_{1-x} \mathrm{P}_{x}\right)_{2}$ (refs 24,25). The $\mathrm{X}-\mathrm{X}$ dimer is fully intact for the whole $\mathrm{CaCo}_{2}\left(\mathrm{Ge}_{1-x} \mathrm{P}_{x}\right)_{2}$ series, and the magnetic properties vary from non-magnetic to antiferromagnetic with no unexpected FM state in the intermediate composition region. In contrast, the $\mathrm{X}-\mathrm{X}$ dimer is fully broken throughout the $\mathrm{BaCo}_{2}\left(\mathrm{Ge}_{1-x} \mathrm{P}_{x}\right)_{2}$ series because of the large size of the $\mathrm{Ba}^{2+}$ ions, and only non-magnetic ground states are observed.

As previously reported, chemical pressure drives $\mathrm{P}-\mathrm{P}$ dimer breaking in $\mathrm{Sr}_{1-x} \mathrm{Ca}_{x} \mathrm{Co}_{2} \mathrm{P}_{2}$, which is associated with a transition from a magnetic cT phase $(x=1)$ to a non-magnetic ucT phase $(x=0)$. A simple explanation for the magnetic transition is 
based on the Zintl concept ${ }^{9,15}$; for example, the singly bonded $\mathrm{P}-\mathrm{P}$ dimer in $\mathrm{CT}$ phases has the electron formal count $(\mathrm{P}-\mathrm{P})^{4-}$, whereas the non-bonded $\mathrm{P}$ in ucT phases has the formal electron count $\mathrm{P}_{2}{ }^{6-}$. Charge redistribution therefore occurs in the $\mathrm{Co}_{2} \mathrm{P}_{2}$ layers during the lattice collapse transition, and changes in the magnetic ground state may be anticipated. A magnetic to non-magnetic transition is observed in $\mathrm{CaFe}_{2} \mathrm{As}_{2}$ when applied pressure induces a lattice collapse ${ }^{17}$. In contrast, $\mathrm{SrCo}_{2}\left(\mathrm{Ge}_{1-x} \mathrm{P}_{x}\right)_{2}$ shows different behaviour: with both endmembers non-magnetic, a FM ground state arises during the dimer breaking that destabilizes the collapsed phase. Within the Zintl concept, the substitution of $\mathrm{P}$ for $\mathrm{Ge}$ in this system does not induce a formal electron count change in the $d$-state because the extra charge contributed by $\mathrm{P}$ doping is consumed through the breaking of the dimer. Therefore the unexpected ferromagnetism cannot be understood as simply being a result of a valence change of the Co.

We speculate that a possible explanation may be found by consideration of differences in the band structure between bonded and non-bonded phases ${ }^{26}$. With the presence of a covalent $\mathrm{Ge}-$ Ge interlayer bond, the chemical potential for $\mathrm{SrCo}_{2} \mathrm{Ge}_{2}$ must be located between the bonding orbitals $(\sigma)$ and antibonding orbitals $\left(\sigma^{\star}\right)$ of the Ge-Ge dimer. In contrast, with the lack of an interlayer $\mathrm{P}-\mathrm{P}$ bond, both the $\sigma$ and $\sigma^{\star}$ orbitals are fully occupied in $\mathrm{SrCo}_{2} \mathrm{P}_{2}$. The $\sigma^{\star}$ band must therefore straddle the chemical potential during the continuous process of dimer breaking in $\mathrm{SrCo}_{2}\left(\mathrm{Ge}_{1-x} \mathrm{P}_{x}\right)_{2}$. A higher density of states at the Fermi level and strong electron correlations therefore might occur when the $\sigma^{\star}$ orbitals and the Co $3 d$ orbitals, which are possibly hybridized with it, are at the chemical potential at intermediate compositions, surpassing the Stoner criterion and driving the material into a band FM state. Further theoretical treatment is needed to validate this proposed mechanism for the unexpected correlation of dimer breaking and the onset of ferromagnetism at a QCP in this system. Finally, we believe that the presence or absence of $\mathrm{X}-\mathrm{X}$ bonding in $\mathrm{ThCr}_{2} \mathrm{Si}_{2}$-type phases, not generally taken as an influence on structure-property relationships in this large family of compounds, requires more careful consideration.

\section{Methods}

Polycrystalline samples were prepared from $1.05 \mathrm{Sr}, 2 x \mathrm{CoP}$ and $2-2 x \mathrm{CoGe}$, as described in ref. 18 , at $950-1,000^{\circ} \mathrm{C}$ for two days. CoP was prepared as in ref. 27 and $\mathrm{CoGe}$ was made from arc-melted $\mathrm{CoGe}_{1.05}$. All the samples were characterized by laboratory X-ray powder diffraction with $\mathrm{Cu} \mathrm{K} \alpha$ radiation (D8 focus, Bruker). Selected samples with $x=0,0.25,0.4,0.5,0.55,0.65,0.8$ and 1.0 were measured by synchrotron X-ray powder diffraction at room temperature using beam line 11-BM at Argonne National Laboratory. We analysed the structure using the program GSAS with EXPGUI (refs 28,29). As the refined $x$ were the same as the nominal $x$ values within $\pm 2 \%$ error, the nominal $x$ values were used for all samples. All physical property characterizations were carried out on a Quantum Design Physical Property Measurement System with a ${ }^{3} \mathrm{He}$ option and a Quantum Design Magnetic Property Measurement System

Received 5 July 2010; accepted 29 October 2010; published online 16 January 2011

\section{References}

1. Stewart, G. R. Non-Fermi-liquid behavior in $d$ - and $f$-electron metals. Rev. Mod. Phys. 73, 797-855 (2001).

2. Stewart, G. R. Addendum: Non-Fermi-liquid behavior in $d$ - and $f$-electron metals. Rev. Mod. Phys. 78, 743-753 (2006).

3. Löhneysen, H. v., Rosch, A., Vojta, M. \& Wölfle, P. Fermi-liquid instabilities at magnetic quantum phase transitions. Rev. Mod. Phys. 79, 1015-1075 (2007).

4. Sokolov, D. A., Aronson, M. C., Gannon, W. \& Fisk, Z. Critical phenomena and the quantum critical point of ferromagnetic $\mathrm{Zr}_{1-x} \mathrm{Nb}_{x} \mathrm{Zn}_{2}$. Phys. Rev. Lett. 96, 116404 (2006).

5. Smith, R. P. et al. Marginal breakdown of the Fermi-liquid state on the border of metallic ferromagnetism. Nature 455, 1220-1223 (2008).
6. Pfleiderer, C., Julian, S. R. \& Lonzarich, G. G. Non-Fermi-liquid nature of the normal state of itinerant-electron ferromagnets. Nature 414, 427-430 (2001).

7. Custers, J. et al. The break-up of heavy electrons at a quantum critical point. Nature 424, 524-527 (2003).

8. Rotter, M., Tegel, M. \& Johrendt, D. Superconductivity at $38 \mathrm{~K}$ in the iron arsenide $\mathrm{Ba}_{1-x} \mathrm{~K}_{x} \mathrm{Fe}_{2} \mathrm{As}_{2}$. Phys. Rev. Lett. 101, 107006 (2008).

9. Hoffmann, R. \& Zheng, C. Making and breaking bonds in the solid state: The thorium chromium silicide $\left(\mathrm{ThCr}_{2} \mathrm{Si}_{2}\right)$ structure. J. Phys. Chem. 89 , 4175-4181 (1985).

10. Just, G. \& Paufler, P. On the coordination of $\mathrm{ThCr}_{2} \mathrm{Si}_{2} \mathrm{BaAl}_{4}$-type compounds within the field of free parameters. J. Alloys Comp. 232, 1-25 (1996).

11. Analytis, J. G. et al. Fermi surface of $\mathrm{SrFe}_{2} \mathrm{P}_{2}$ determined by the de Haas- van Alphen effect. Phys. Rev. Lett. 103, 076401 (2009).

12. Coldea, A. I. et al. Topological change of the Fermi surface in ternary iron pnictides with reduced $c / a$ ratio: A de Haas-van Alphen study of $\mathrm{CaFe}_{2} \mathrm{P}_{2}$ Phys. Rev. Lett. 103, 026404 (2009).

13. Yildirim, T. Strong coupling of the Fe-spin state and the As-As hybridization in iron-pnictide superconductors from first-principle calculations. Phys. Rev. Lett. 102, 037003 (2009).

14. Huhnt, C., Schlabitz, W., Wurth, A., Mewis, A. \& Reehuis, M. First-order phase transitions in $\mathrm{EuCo}_{2} \mathrm{P}_{2}$ and $\mathrm{SrNi}_{2} \mathrm{P}_{2}$. Phys. Rev. B 56, 13796-13804 (1997).

15. Reehuis, M., Jeitschko, W., Kotzyba, G., Zimmer, B. \& Hu, X. Antiferromagnetic order in the $\mathrm{ThCr}_{2} \mathrm{Si}_{2}$ type phosphides $\mathrm{CaCo}_{2} \mathrm{P}_{2}$ and $\mathrm{CeCo}_{2} \mathrm{P}_{2}$. J. Alloys Comp. 266, 54-60 (1998).

16. Chefki, M. et al. Pressure-induced transition of the sublattice magnetization in $\mathrm{EuCo}_{2} \mathrm{P}_{2}$ : Change from local moment $\mathrm{Eu}(4 f)$ to itinerant $\mathrm{Co}(3 d)$ magnetism. Phys. Rev. Lett. 80, 802-805 (1998).

17. Canfield, P. C. et al. Structural, magnetic and superconducting phase transitions in $\mathrm{CaFe}_{2} \mathrm{As}_{2}$ under ambient and applied pressure. Physica $\mathrm{C}$ 469, 404-412 (2009).

18. Jia, S., Williams, A. J., Stephens, P. W. \& Cava, R. J. Lattice collapse and the magnetic phase diagram of $\mathrm{Sr}_{1-x} \mathrm{Ca}_{x} \mathrm{Co}_{2} \mathrm{P}_{2}$. Phys. Rev. B 80, 165107 (2009).

19. Reehuis, M. \& Jeitschko, W. Structure and magnetic properties of the phosphides $\mathrm{CaCo}_{2} \mathrm{P}_{2}$ and $\mathrm{LnT}_{2} \mathrm{P}_{2}$ with $\mathrm{ThCr}_{2} \mathrm{Si}_{2}$ structure and LnTP with $\mathrm{PbFCl}$ structure $(\mathrm{Ln}=$ lanthanoids, $\mathrm{T}=\mathrm{Fe}, \mathrm{Co}, \mathrm{Ni})+$. J. Phys. Chem. Solids 51, 961-968 (1990).

20. Arrott, A. Criterion for ferromagnetism from observations of magnetic isotherms. Phys. Rev. 108, 1394-1396 (1957).

21. Moriya, T. Spin Fluctuations in Itinerant Electron Magnetism (Springer, 1985)

22. Millis, A. J. Effect of a nonzero temperature on quantum critical points in itinerant fermion systems. Phys. Rev. B 48, 7183-7196 (1993).

23. Mydosh, J. A. Spin Glass: An Experimental Introduction (Taylor and Francis, 1993).

24. Pawina, J. Bachelor Thesis, Princeton Univ. (2010).

25. Siggelkow, L., Hlukhyy, V. \& Fässler, T. F. Synthesis, structure and chemical bonding of $\mathrm{CaCo}_{2} \mathrm{Si}_{2}$ and $\mathrm{BaCo}_{2} \mathrm{Ge}_{2}$ - two new compounds with $\mathrm{ThCr}_{2} \mathrm{Si}_{2}$ structure type. Z. Anorg. Allg. Chem. 636, 378-384 (2010).

26. Hoffmann, R. Solids and Surfaces: A Chemist's View of Bonding in Extended Strutures (VCH, 1988).

27. McQueen, T. M. et al. Intrinsic properties of stoichiometric LaFePO. Phys. Rev. B 78, 024521 (2008).

28. Larson, A. C. \& Von Dreele, R. B. General Structure Analysis System (GSAS). Report No. 86 (Los Alamos National Laboratory LAUR, 2000).

29. Toby, B. H. EXPGUI, a graphical user interface for GSAS. J. Appl. Crystallogr. 34, 210-213 (2001)

\section{Acknowledgements}

The authors thank J. Xiong and D. X. Qu for experimental assistance, as well as N. Ni for helpful discussion. The work at Princeton was supported by the US Department of Energy, Division of Basic Energy Sciences, Grant No. DE-FG02-98ER45706. Use of the Advanced Photon Source at Argonne National Laboratory was supported by the US Department of Energy, Office of Science, Office of Basic Energy Sciences, under Contract No. DE-AC02-06CH11357.

\section{Author contributions}

P.J. and S.J. synthesized the materials. M.R.S. and B.H.T. performed the synchrotron XRD. S.J., J.G.C. and N.P.O. performed the thermodynamic measurements. S.J., P.J. and R.J.C. analysed the data. S.J. and R.J.C. wrote the paper. R.J.C. designed the study.

\section{Additional information}

The authors declare no competing financial interests. Reprints and permissions information is available online at http://npg.nature.com/reprintsandpermissions. Correspondence and requests for materials should be addressed to R.J.C. 